\title{
BMJ Open Dislocated distal radial fractures in adult patients: 4 weeks versus 6 weeks of cast immobilisation following reduction, a multicentre randomised controlled trial, study protocol
}

\author{
Eva A K van Delft, ${ }^{1}$ Frank W Bloemers, ${ }^{2}$ Nico L Sosef, ${ }^{3} \mathrm{H}$ J Bonjer, ${ }^{4}$ \\ Niels W L Schep, ${ }^{5}$ Jefrey Vermeulen ${ }^{5}$
}

To cite: van Delft EAK, Bloemers FW, Sosef NL, et al. Dislocated distal radial fractures in adult patients: 4 weeks versus 6 weeks of cast immobilisation following reduction, a multicentre randomised controlled trial, study protocol. BMJ Open 2019;9:e026540. doi:10.1136/ bmjopen-2018-026540

- Prepublication history for this paper is available online. To view these files, please visit the journal online (http://dx.doi. org/10.1136/bmjopen-2018026540).

Received 22 0ctober 2018 Revised 18 December 2018 Accepted 21 December 2018

Check for updates

(c) Author(s) (or their employer(s)) 2019. Re-use permitted under CC BY-NC. No commercial re-use. See rights and permissions. Published by BMJ.

For numbered affiliations see end of article.

Correspondence to Mrs Eva A K van Delft; e.vandelft@vumc.nl

\section{ABSTRACT}

Introduction Up to $30 \%$ of patients with a dislocated distal radial fracture (DRF) treated with closed reduction and cast immobilisation suffer from long-term functional restrictions. It remains unclear, whether duration of cast immobilisation influences functional outcome. The aim of this study is to evaluate whether the functional outcome of dislocated DRFs could be improved by shortening the period of immobilisation.

Methods and analysis A single blinded multicentre randomised controlled trial is initiated. Four weeks of plaster cast immobilisation is compared with six week plaster cast immobilisation in adult patients with adequate reduced DRFs. Primary outcome parameters are functional outcome measured with the Patient Rated Wrist Evaluation after 1 year of follow-up (FU). Secondary outcomes are: Disability of Arm, Shoulder and Hand Score after 1 year, 36-Item Short Form Health Survey after 1 year, functional outcome earlier in FU (6 weeks, 12 weeks and 6 months), range of motion, pain level and complications: number of re-interventions, secondary dislocation, delayed and non-union.

Ethics and dissemination The medical ethical committee VUmc approved the study protocol (2018.004, NL62861.029.17). The expectation of this study is that a shorter duration of plaster cast immobilisation is beneficial. This risk of specific complications is low and generally similar in both treatment options. FU is standardised according to current trauma guidelines. Present literature indicates that both treatment options that are used within this study are accepted protocols for treatment of dislocated DRFs. This trial will provide Level-I evidence for the comparison of functional outcome between the two treatment options for dislocated DRFs. Results of this study are expected to be published as a prospective, multicentre, randomised controlled trial article in 2021.

Trial registration The Netherlands National Trial Register: NTR 6600, ABR: NL62861.029.17. Medical Ethical Committee VUmc registration number: 2018.004 .

\section{INTRODUCTION}

Distal radial fractures (DRF) are common fractures and account for up to $20 \%$ of all extremity fractures. ${ }^{1}$ Most of these patients

\section{Strengths and limitations of this study}

- This study is designed as a single blinded study, it was not possible to perform this study in a double-blinded setting.

- This multicentre study will be carried out in two hospitals in the Netherlands.

- This study uses validated outcomes (Patient Rated Wrist Evaluation, Quick Disability of Arm and Shoulder, 36-Item Short Form Health Survey).

- This study uses both statistical as well as minimal clinical important difference.

This trial will provide level-I evidence for the period of immobilisation in reduced distal radial fractures.

can be treated non-operatively in a plaster with excellent functional results. ${ }^{23}$ Nevertheless, up to $30 \%$ of patients with a dislocated DRF suffer from long-term functional restrictions following conservative treatment as neuropathy, arthrosis and stiffness. ${ }^{4}$

Unstable DRFs are liable to dislocate within the first 2 weeks, only $7-8 \%$ dislocate after this time and none after 6 weeks. ${ }^{5-7}$ Therefore, a period of up to 6 weeks of immobilisation is advised, although, this is still a matter of debate in literature. ${ }^{89}$

Two prospective studies of patients with dislocated and reduced DRF showed that a shorter immobilisation period was safe, without increased numbers of (re) dislocation of the fracture. ${ }^{810}$ Besides, the outcome seemed to be better on the long-term, in terms of wrist motion and grip strength. Unfortunately, these studies were non-randomised and conducted in heterogeneous groups of patients suffering both non-dislocated and dislocated fractures. Obviously, the best treatment of reduced DRF will be short, safe and will lead to an early return of 


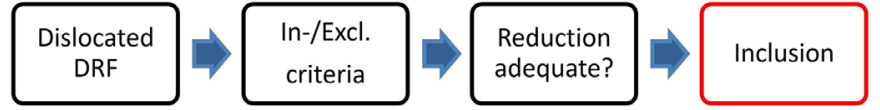

Figure 1 Inclusion procedure.

function. To assess whether reduction of the immobilisation period with 2 weeks will lead to better functional outcome, a multicentre randomised controlled trial will be conducted.

The patient reported functional outcome after 1 year will be assessed using validated instruments: the Patient Rated Wrist Evaluation (PRWE), the Quick Disability of Arm and Shoulder (DASH) and 36-Item Short Form Health Survey (SF-36). ${ }^{11-13}$ Other outcome measures will be the functional outcome earlier in follow-up (FU), the amount of pain measured on the visual analogue sqale (VAS), number of secondary dislocations, number of re-interventions, range of motion, delayed and non-union and complex regional pain syndrome (CRPS).

The aim of this trial is to compare the results of 4 weeks of cast immobilisation with 6 weeks of cast immobilisation in closed and adequate reduced DRF. Usually, an immobilisation period of 5 or 6 weeks is preferred as non-operative treatment of closed and adequate reduced DRF. Despite the minimal evidence in literature, this immobilisation period can be questioned. A randomised clinical trial with sufficient power will be needed to provide scientific support for a preferred treatment strategy for reduced DRF.

\section{METHODS AND ANALYSIS}

This study will be conducted as a prospective single blinded multicentre randomised clinical trial in two large teaching hospitals. In this study, 4 weeks of plaster immobilisation will be compared with 6 weeks of plaster immobilisation (figures 1 and 2). The methods of this study protocol are comparable to a previous published article comparing 3 weeks of cast immobilisation to 5 weeks of cast immobilisation in adult patients with non-displaced DRF. ${ }^{1415}$ Patients will be treated in a lower arm cast in neutral position. ${ }^{16}$ Following immobilisation, treatment will be the same for both groups, in which additional physiotherapy after removal of the cast is advised and exercises to train wrist function will be given. As extra structured advise programmes may cause no extra benefit for the patient, this was not generally prescribed. ${ }^{17}$

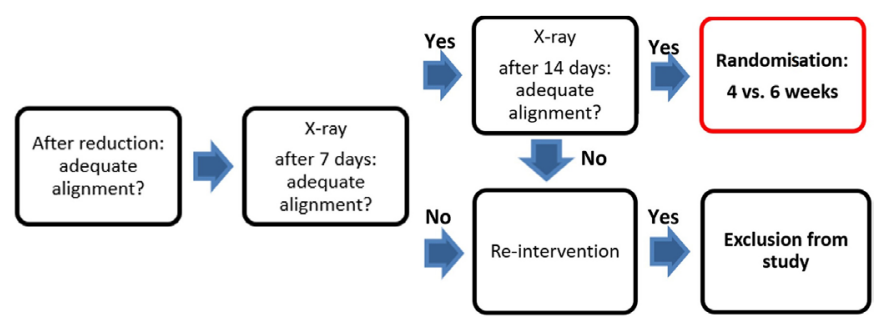

Figure 2 Control of alignment and randomisation procedure.
However, during FU visits, patients will be asked if they were treated by a physiotherapist. If this is the case, details on the number of sessions per week and the total number of weeks the patient received physiotherapy, will be collected.

\section{Participants}

Evaluation of eligible patients will take place either at the emergency department (ED) or at the outpatient department. They will receive written information and a consent form from the attending physician, the clinical investigator or a research assistant. Patients will be eligible if they follow the inclusion and exclusion criteria.

\section{Inclusion criteria}

1. Age $>18$ years.

2. Primary dislocated unilateral DRF.

3. Independent for activities of daily living.

\section{Exclusion criteria}

1. Fracture of the contralateral wrist.

2. Ipsilateral fractures proximal of the DRF.

3. Pre-existent abnormalities or functional deficits of the fractured wrist that influences the patient reported function of the wrist.

4. Open fractures.

5. Language ability to understand the Dutch patient information and questionnaires.

Patients will only be able to participate if closed reduction of the DRF is adequate. The indication for reduction will be set, using the Lidström criteria for misalignment. ${ }^{18}$ Patients will only be able to participate in this study if reduction is performed successfully. Successful reduction will be classified as: radial shortening $<3 \mathrm{~mm}$, dorsal tilt $<10^{\circ}$ or intra-articular step-off $<2 \mathrm{~mm}$, according the guidelines of the American Academy of Orthopaedic Surgeons. ${ }^{19}$

After providing informed consent, eligible patients will be randomised after 2 weeks when the fracture has proven to be stable. An independent research assistant will perform concealed permuted block randomisation using a computer-generated randomisation schedule after stratification for fracture type, gender and age. Allocation will be at random in four blocks. To prevent bias, stratification by age (younger and older than 60 years) and gender will be performed (table 1).

Randomisation between another 2 or 4 weeks cast immobilisation will be performed to complete a total of 4 and 6 weeks of cast immobilisation, respectively. Randomisation will occur after informed consent.

The primary outcome measure of this study is PRWE after 1 year. ${ }^{11}$ The secondary outcome measures are The QuickDASH score after 1 year ${ }^{12}$; the SF-36 Healthy Survey after 1 year ${ }^{13}$; functional outcome after 8 weeks, 3 months and 6 months; range of motion; pain level after 8 weeks, 3 months, 6 months and 1 year; Lidström score ${ }^{18}$ and fracture-related complications: secondary dislocation after 


\begin{tabular}{|c|c|}
\hline $\begin{array}{l}\text { Stratification by gender and } \\
\text { age }\end{array}$ & \\
\hline Patient characteristics & Randomisation \\
\hline List 1 Male $<60$ years of age & $\begin{array}{l}\text { ABAB AABB ABBA BABA } \\
\text { BAAB }\end{array}$ \\
\hline List 2 Male $>60$ years of age & $\begin{array}{l}\text { BAAB BBAA ABAB AABB } \\
\text { ABBA }\end{array}$ \\
\hline $\begin{array}{l}\text { List } 3 \text { Female }<60 \text { years of } \\
\text { age }\end{array}$ & $\begin{array}{l}\text { AABB ABBA BAAB BBAA } \\
\text { BABA }\end{array}$ \\
\hline $\begin{array}{l}\text { List } 4 \text { Female }>60 \text { years of } \\
\text { age }\end{array}$ & $\begin{array}{l}\text { ABBA BABA ABAB AABB } \\
\text { AABB }\end{array}$ \\
\hline
\end{tabular}

A, 4 weeks; B, 6 weeks.

cast removal, number of re-interventions, delayed and non-unions and CRPS.

PRWE score is the most responsive instrument for evaluating the outcome in patients with DRF. The PRWE is a validated 15-item (scored 1-10), self-reported questionnaire designed to help describe the disability experienced by people with disorders of the wrist and also to monitor changes in symptoms and function over time. Scores will be transformed to a $0-100$ score. $^{11}$ A higher score will indicate greater disability.

The DASH outcome measure is a validated 30-item, self-reported questionnaire designed to help describe the disability experienced by people with upper-limb disorders and also to monitor changes in symptoms and function over time. ${ }^{19}$ The QuickDASH is a shortened version of the DASH Outcome Measure. Instead of 30 items, the QuickDASH uses 11 items (scored 1-5) to measure physical function and symptoms in people with any or multiple musculoskeletal disorders of the upper limb. At least, 10 of the 11 items must be completed for a score to be calculated. The scores will be transformed to a $0-100$ scale for easy comparison. A higher score will indicate greater disability. ${ }^{12}$

The SF-36 is a validated 36-item, self-reported questionnaire designed to describe the quality of life. The score consists eight subgroups: vitality, mental health social role, emotional role, physical role, general health, bodily pain and physical functioning. The subgroups are transformed to a $0-100$ scale. The lower the score will be, the more disability, an higher score will indicate less disability. ${ }^{13}$

After inclusion, all patients will be followed for 1 year in total. Clinical assessments will occur at the time of admission (ED), 1 week (3-10 day window), 2 weeks (11-18 day window), 4weeks (24-32 day window) or 6 weeks (5-7 week window), 3 months (11-15 week window), 6 months (5-7 month window) and 12 months (11-14 month window) after inclusion.

At each FU visit, the research coordinator or research assistant will ascertain patient status (ie, secondary
FOUR WEEKS OF PLASTER CAST IMMOBILISATION

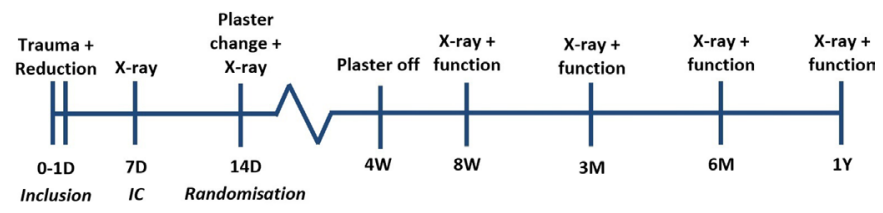

SIX WEEKS OF PLASTER CAST IMMOBILISATION

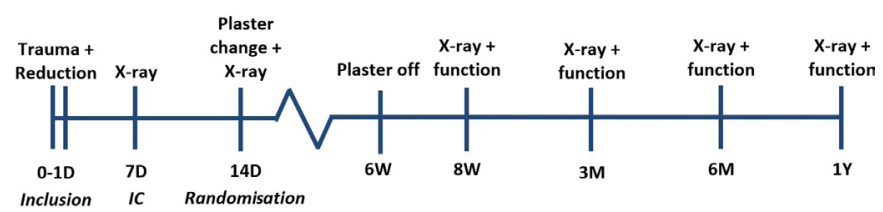

Figure 3 Follow-up scheme 4 versus 6 weeks of plaster cast immobilisation.

interventions, adverse events/complications, deaths) and will verify information within medical records. All adverse events will be addressed to the principal investigator.

At each FU visit, the patients will be asked to indicate the actual pain level on a VAS. Patients will also be asked if they have any complaints of their treatment and will be asked if they are currently treated by a physical therapist. At each visit from 8 weeks onwards, the range of motion of the wrist will be measured using a goniometer, according to the reference values for joint range of motion published by the American Academy of Orthopaedic Surgeons. ${ }^{20}$ In addition, patients will be asked to complete the questionnaires relating to disability (QuickDASH score, PRWE, SF-36).

Plain X-rays of the wrist will be made at the time of presentation in the hospital (ED), after 1 and 2 weeks, 4 or 6 weeks and at the FU visit after 8 weeks, 3 months, 6 months and 1 year. The X-ray at 1 year will be taken in order to determine the grade of degenerative joint changes. Time to define the presence of a delayed- or non-union will be at 3 or 6 months (figures 1-3).

The primary outcome will be the PRWE Score, of which the minimal clinically important difference is 11.5 points. The SD of the PRWE is 14.0. ${ }^{21}$ Based on a difference of 11.5 points, the sample size of 27 patients per treatment group is calculated with a power (1- $\beta$ ) of $80 \%$ and a type I error $(\alpha)$ of $5 \%$, allowing for $10 \%$ dropout. In this study, we decided to include 45 patients per treatment group. To allow a $10 \%$ dropout in this study, in total 100 patients will be included.

Data from the demographic data collection and the outcome parameters will be cleaned blindly from the treatment data. Data are presented as mean scores with 95\% confidence intervals. The analysis of this study will be carried out according to the intention-to-treat principle, that is, the patients will remain in the group they will be randomly allocated to at baseline. Analysis of functional outcome will be assessed using repeated-measures analysis of variance (GLM 4) with the time as the within-group factor and the treatment as the between-group factor. Post hoc analysis will be performed on the time of randomisation. Group comparisons at the different time 
points will be made only when the overall repeated-measures tests are statistically significant. All scores will be tested for normality using the Kolmogorov-Smirnov test. Parametric variables will be compared using the Student's t-test, while non-parametric and ordinal variables will be compared using the Mann-Whitney U statistic. Nominal variables will be compared across independent groups using the chi-squared test or Fisher's exact test. Homogeneity of variance will be assessed using Levene's test. Also, a multiple regression will be performed. SPSS statistical software (version 24.0) will be used for the analysis, in which two-tailed $\mathrm{p}$ value $<0.05$ will be considered significant.

\section{Patient and public involvement}

Patients were not involved in the research process. Although, during the time span the study is carried out, participants receive an annually update on the progress of the study by a specially developed newsletter.

\section{ETHICS AND DISSEMINATION}

Present literature indicates that 4 weeks of immobilisation as well as 6 weeks of immobilisation are both accepted protocols for treatment of dislocated DRF. In daily practise, a 6 weeks immobilisation period is mostly used. To assess the clinical controversy on this duration of treatment, this study is initiated.

The studies done for assessing the immobilisation periods of DRF have their limitations of using non-validated outcome score lists, which makes it impossible to conclude with certainty shorter immobilisation periods of DRF are preferred.

The expectation of this study is that a shorter duration of plaster cast immobilisation is beneficial for the patients. This risk of specific complications is low and generally similar in both treatment options.

The Medical Ethical Committee VUmc has approved the study protocol (2018.004).

This trial will provide Level-1 evidence for the comparison of functional outcome between the two treatment options for dislocated DRF. Results of this study are expected to be published as a prospective, multicentre, randomised controlled trial article in 2021.

\section{Author affiliations}

${ }^{1}$ Amsterdam UMC, Vrije Universiteit Amsterdam, Department of Trauma Surgery, Amsterdam Movement Sciences, the Netherlands

${ }^{2}$ Amsterdam UMC, Vrije Universiteit Amsterdam, Department of Trauma Surgery, the Netherlands

${ }^{3}$ Spaarne Gasthuis, Department of Trauma Surgery, Haarlem, the Netherlands ${ }^{4}$ Amsterdam UMC, Vrije Universiteit Asmterdam, Department of Surgery, the Netherlands

${ }^{5}$ Maasstad Ziekenhuis, Department of Trauma Surgery, Rotterdam, the Netherlands

Contributors EAKvD: Study development and writing study protocol. Clinical investigator in Amsterdam UMC, VU Medical Center. FWB: Project leader, corrections of study protocol. NLS: First clinical investigator in Spaarne Gasthuis. HJB: Head of Department of Surgery, Amsterdam UMC, VU Medical Center. Approval of the study protocol. NWLS: Study development. JV: Initial first clinical investigator in Spaarne Gasthuis, study development and corrections of study protocol.
Funding The authors have not declared a specific grant for this research from any funding agency in the public, commercial or not-for-profit sectors.

Competing interests None declared.

Patient consent for publication Not required.

Ethics approval The Medical Ethics Committee VUmc, The Netherlands (2018.004) has approved the study protocol.

Provenance and peer review Not commissioned; externally peer reviewed.

Open access This is an open access article distributed in accordance with the Creative Commons Attribution Non Commercial (CC BY-NC 4.0) license, which permits others to distribute, remix, adapt, build upon this work non-commercially, and license their derivative works on different terms, provided the original work is properly cited, appropriate credit is given, any changes made indicated, and the use is non-commercial. See: http://creativecommons.org/licenses/by-nc/4.0/.

\section{REFERENCES}

1. Meena S, Sharma P, Sambharia AK, et al. Fractures of distal radius: an overview. J Family Med Prim Care 2014;3:325-32.

2. Cooney WP. Management of Colles' fractures. J Hand Surg Br 1989;14:137-9.

3. McQueen M, Caspers J. Colles fracture: does the anatomical result affect the final function? J Bone Joint Surg Br 1988;70:649-51.

4. Cooney WP, Dobyns JH, Linscheid RL. Complications of Colles' fractures. J Bone Joint Surg Am 1980;62:613-9.

5. Solgaard S. Early displacement of distal radius fracture. Acta Orthop Scand 1986;57:229-31.

6. Abbaszadegan $\mathrm{H}$, von Sivers $\mathrm{K}$, Jonsson $\mathrm{U}$. Late displacement of Colles' fractures. Int Orthop 1988;12:197-9.

7. Solgaard S. Function after distal radius fracture. Acta Orthop Scand 1988;59:39-42.

8. McAuliffe TB, Hilliar KM, Coates CJ, et al. Early mobilisation of Colles' fractures. J Bone Joint Surg Br 1987;69:727-9.

9. Christensen OM, Christiansen TG, Krasheninnikoff M, et al. Length of immobilisation after fractures of the distal radius. Int Orthop 1995;19:26-9.

10. Vang Hansen F, Staunstrup H, Mikkelsen S. A comparison of 3 and 5 weeks immobilization for older type 1 and 2 Colles' fractures. J Hand Surg Br 1998;23:400-1.

11. MacDermid JC, Turgeon T, Richards RS, et al. Patient rating of wrist pain and disability: a reliable and valid measurement tool. J Orthop Trauma 1998;12:577-86.

12. Gummesson C, Ward MM, Atroshi I. The shortened disabilities of the arm, shoulder and hand questionnaire (QuickDASH): validity and reliability based on responses within the full-length DASH. BMC Musculoskelet Disord 2006;7:7-44.

13. Aaronson NK, Muller M, Cohen PD, et al. Translation, validation, and norming of the Dutch language version of the SF-36 Health Survey in community and chronic disease populations. J Clin Epidemiol 1998;51:1055-68.

14. Bentohami A, de Korte N, Sosef N, et al. Study protocol: nondisplaced distal radial fractures in adult patients: three weeks vs. five weeks of cast immobilization: a randomized trial. BMC Musculoskelet Disord 2014;15:24.

15. Bentohami A, van Delft E, Vermeulen J, et al. Non- or minimally displaced distal radial fractures in adult patients: three weeks versus five weeks of cast immobilization - a randomized controlled trial. J Wrist Surg 2019;08:043-8.

16. Handoll HHG, Madhok R. Conservative interventions for treating distal radial fractures in adults. Cochrane Database Syst Rev 2003;60:CD000314.

17. Bruder AM, Shields N, Dodd KJ, et al. A progressive exercise and structured advice program does not improve activity more than structured advice alone following a distal radial fracture: a multicentre, randomised trial. J Physiother 2016;62:145-52.

18. Lidström A. Fractures of the distal end of the radius. A clinical and statistical study of end results. Acta Orthop Scand Suppl 1959;41:1-118.

19. AAOS. The treatment of distal radius fractures guideline and evidence report. 2009.

20. Greene W, Heckman JD. The clinical measurement of joint motion. Rosemont, IL: American Academy of Orthopaedic Surgeons, 1994.

21. Walenkamp MM, de Muinck Keizer RJ, Goslings JC, et al. The minimum clinically important difference of the patient-rated wrist evaluation score for patients with distal radius fractures. Clin Orthop Relat Res 2015;473:3235-41. 